\title{
Biofilms of Candida spp. from the ocular conjunctiva of horses with reduced azole susceptibility: a complicating factor for the treatment of keratomycosis?
}

\author{
Raimunda Sâmia Nogueira Brilhante, ${ }^{*}$ Paula Vago Bittencourt, $†$ Débora de Souza Collares Castelo-Branco,* \\ Glaucia Morgana de Melo Guedes, ${ }^{*}$ Jonathas Sales de Oliveira, ${ }^{*}$ Lucas Pereira Alencar, ${ }^{*}$ \\ Rossana de Aguiar Cordeiro, ${ }^{*}$ Mariana Pinheiro, $\uparrow$ Evilázio Fernandes Nogueira-Filho, $\dagger$ \\ Waldemiro de Aquino Pereira-Neto, $屯$ José Júlio Costa Sidrim* and Marcos Fábio Gadelha Rocha*, $\dagger$ \\ *Department of Pathology and Legal Medicine, Graduate Program in Medical Microbiology, Specialized Medical Mycology Center, School of Medicine, Fed- \\ eral University of Ceará, Fortaleza, CE, Brazil; †Postgraduate Program in Veterinary Sciences, School of Veterinary Medicine, State University of Ceará, \\ Fortaleza, CE, Brazil; and $\$$ Department of Transport Engineering, Federal University of Ceará, Fortaleza, CE, Brazil
}

Address communications to:

R. S. N. Brilhante

Tel.: +558532951736

Fax: 55 (85) 3295-1736

e-mail: brilhante@ufc.br

\begin{abstract}
Objectives This study aimed to assess the biofilm-forming ability of Candida spp. from the ocular conjunctiva of horses and to investigate the antifungal susceptibility of these biofilms.

Procedures Initially, the biofilm-forming ability of 15 strains was assessed by crystal violet staining, which reveals the fungal biomass adhered to the polystyrene plates, and scanning electron microscopy. Then, the minimum inhibitory concentrations (MICs) of amphotericin B, fluconazole, itraconazole, and caspofungin were initially determined against strains in planktonic form. Afterward, antifungal susceptibility of mature biofilms was evaluated by exposing them to $10 \times$ MIC and $50 \times$ MIC of the tested drugs, followed by the assessment of their metabolic activity, using the oxidoreduction indicator XTT. Results were analyzed through ANOVA and Tukey's post-test, and $P$ values below $5 \%$ led to significant conclusions.

Results Eight strains produced biofilms and were classified as strong (1/15), moderate $(3 / 15)$ and weak (4/15) producers, according to the amount of crystal violet retained by the adhered fungal biomass. Biofilm metabolic activity of one $C$. tropicalis did not decrease after exposure to the tested antifungals, while biofilm metabolic activity of five strains was reduced by amphotericin B, but not the other drugs. One $C$. parapsilosis sensu stricto and one $C$. glabrata showed significant reduction in biofilm metabolic activity after exposure to fluconazole, itraconazole, and caspofungin, but not amphotericin B.

Conclusions The results demonstrate that Candida from the ocular conjunctiva of horses can pose as a risk to animal health as they are capable of forming biofilms, which are commonly involved in fungal keratitis.
\end{abstract}

Key Words: antifungal susceptibility, biofilm, Candida spp, horses, microbiota, yeasts

\section{INTRODUCTION}

Fungi are normally present in places where horses inhabit and are part of their corneal and conjunctival microbiota. ${ }^{1}$ The conjunctival microbiota of horses is variable, depending on the animal's age, season of the year, geographic location, and presence of bacteria and fungi, including Candida spp., in the environment where animals are raised.
The prevalence of these yeasts varies from 3 to $20 \%^{2-4}$ and they can act as ocular pathogens, when there are adequate conditions in the host. ${ }^{1,2,6}$ It is known that the presence of fungi in the conjunctival microbiota, including Candida spp., is a risk factor for the development of keratomycosis. ${ }^{1,4}$ Moreover, Candida spp. have been reported as the third most common etiological agent of equine keratomycosis. ${ }^{7}$ 
The pathogenicity of Candida species is mediated by several virulence factors, including the ability to evade host defenses, production of hydrolytic enzymes, and the ability to adhere to the host tissues, especially mucous membranes, and medical devices, such as central venous catheters or prostheses, where these yeasts can form biofilms. ${ }^{8}$ Biofilms are defined as well-structured microbial communities that are adhered to a surface, and embedded in an extracellular polymeric matrix. ${ }^{9}$ The advantages of living within a biofilm include protection against the environment, resistance to physical and chemical stresses, metabolic cooperation, and joint regulation of gene expression for the associated microorganisms. ${ }^{10}$ It is important to highlight that biofilm growth is often involved in the development of Candida keratitis, ${ }^{11}$ and these biofilms are commonly resistant to antifungal agents, thus, becoming inherently difficult to treat. ${ }^{12}$

Therefore, the aim of this study was to assess the biofilm-forming ability of Candida spp. from the ocular conjunctiva of horses and to investigate the antifungal susceptibility of these biofilms.

\section{MATERIALS AND METHODS}

\section{Microorganisms}

Fifteen strains of Candida (four C. tropicalis, three C. parapsilosis sensu stricto, two C. glabrata, one C. rugosa, one C. famata, one C. ciferrii, one C. guilliermondii, one C. catenulata, and one C. krusei) obtained from the ocular conjunctiva of clinically healthy horses from the state of Ceará, Northeastern Brazil, were used in this study. The strains belong to the culture collection of the Specialized Medical Mycology Center (CEMM) of the Federal University of Ceará, where they are stored in saline solution at $4{ }^{\circ} \mathrm{C}$. The strains were recovered by seeding onto potato agar. Then, they were subcultured on a chromogenic medium to assure the purity of the stocks, followed by phenotypical identification based on morphological and biochemical features. This study was approved by the ethics committee of the State University of Ceará (UECE), protocol number 12776861-0.

\section{Biofilm formation}

The 15 studied strains were analyzed for their ability to form biofilm, through the method described by Chatzimoschou et al. ${ }^{13}$ with some modifications. Initially, the strains were grown on Sabouraud agar for $48 \mathrm{~h}$ at $30{ }^{\circ} \mathrm{C}$. Then, they were transferred to Sabouraud broth and incubated under agitation $(150 \mathrm{rpm})$ for $24 \mathrm{~h}$. After this period, the tubes containing the isolates were centrifuged (300 rpm, $10 \mathrm{~min}$ ) and the supernatant was discarded. The resulting pellets were washed with sterile PBS, twice, followed by another centrifugation step. Then, the fungal pellets were resuspended in RPMI medium and the inoculum turbidity was adjusted to 0.5 on McFarland scale, which contains approximately 1 to $5 \times 10^{6} \mathrm{cfu} / \mathrm{mL}$. Then, $100-\mu \mathrm{L}$ aliquots of each Candida inoculum were incubated in the wells of a 96-well flat-bottom polystyrene plate, in triplicate, and kept under agitation for $48 \mathrm{~h}(150 \mathrm{rpm})$ at $37^{\circ} \mathrm{C}$. Yeast-free negative control wells were included in the analysis, which contained only RPMI medium. C. albicans ATCC 10231 is a well-known biofilm producer and was used as positive control for biofilm production. After incubation, the supernatant from the wells was carefully aspirated and washed three times with PBS-Tween. The wells were washed with $100 \mu \mathrm{L}$ of $100 \%$ methanol. After drying, $100 \mu \mathrm{L}$ of a $0.3 \%$ crystal violet solution was added to each well and incubated for $20 \mathrm{~min}$. Afterward, crystal violet was removed and the wells were washed twice with $200 \mu \mathrm{L}$ of sterile-distilled water. Then, $150 \mu \mathrm{L}$ of a $33 \%$ acetic acid solution was added to each stained well and allowed to act for $30 \mathrm{~s}$. Immediately after this period, the volume from each well was transferred to the well of another 96-well flat-bottom polystyrene plate and read with a spectrophotometer at $590 \mathrm{~nm}$. Crystal violet dyes microbial structures and extracellular polymeric substances adhered to the 96-well polystyrene plates, and it is used to quantify biofilm biomass. Higher absorbance values at $590 \mathrm{~nm}$ indicate greater crystal violet retention, hence, thicker and denser biofilm biomass. ${ }^{14}$

The cut-off value (ODc) for biofilm formation was defined as three standard deviations above the mean absorbance of the yeast-free negative control. ${ }^{14}$ The strains were, then, classified as nonbiofilm producers, when OD590 $\mathrm{nm} \leq$ ODc, weak biofilm producers, when ODc $<$ OD590 nm $\leq 2 \times$ ODc, moderate biofilm producers, when $2 \times$ ODc $<$ OD590 $\mathrm{nm} \leq 4 \times$ ODc, or strong biofilm producers, when OD590 $\mathrm{nm}>4 \times$ ODc. $^{14}$ The ODc value obtained in these analyses was 0.477 .

\section{Scanning electron microscopic study of Candida spp. biofilms}

The biofilms were analyzed by scanning electron microscopy (SEM) according to Wang et al. ${ }^{15}$ with modifications. Biofilms were formed directly on sterile glass slides covered with poly-l-lysine, using the method described above. After incubation, biofilm supernatants were removed and the slides were washed twice with cacodylate buffer $(0.15 \mathrm{M})$. Biofilms were then covered with glutaraldehyde $(2.5 \%$ in cacodylate buffer $0.15 \mathrm{M})$ and incubated at $4{ }^{\circ} \mathrm{C}$ overnight. After incubation, the biofilms were washed twice with cacodylate buffer for $5 \mathrm{~min}$ and the slides were dehydrated in an ascending ethanol series (50, 70, 80, 95 and 100\%) twice, for 10 min each time. The slides were dried at room temperature and covered with hexamethyldisilazane (HMDS) during $30 \mathrm{~min}$, after which the HDMS was removed and the slides were dried overnight, coated with $10 \mathrm{~nm}$ of gold (Emitech Q150T), and observed with an FEI Inspect S50 scanning electron microscope, in high vacuum mode at $15 \mathrm{kV}$. The images were processed with the Photoshop software (Adobe Systems, San José, Calif.). 
Determination of the antifungal minimum inbibitory concentration against planktonic Candida spp. cells

The minimum inhibitory concentration (MIC) for the antifungal drugs against the strains of Candida in freeflowing planktonic growth was determined by the broth microdilution method, as standardized by the Clinical and Laboratory Standards Institute. ${ }^{16}$ Amphotericin B (Sigma, USA), itraconazole (Janssen Pharmaceutica, Belgium), fluconazole (Pfizer, Brazil) and caspofungin (Sigma, USA) were prepared according to CLSI guidelines. ${ }^{16}$ Amphotericin $\mathrm{B}$ and itraconazole were initially diluted with DMSO, while fluconazole and caspofungin were diluted with water. ${ }^{16}$ Even though amphotericin B and caspofungin are not commonly used to treat fungal infections in animals, they were tested as representatives of the polyene and echinocandin class of antifungal drugs, respectively. Additionally, fluconazole and itraconazole were included as representatives of the azole antifungals because they are the most commonly used antifungal drugs in veterinary practice. The tested concentration ranges were $0.125-64 \mu \mathrm{g} / \mathrm{mL}$ for fluconazole and $0.03125-16 \mu \mathrm{g} / \mathrm{mL}$ for amphotericin B, itraconazole, and caspofungin. Each test was performed in duplicate. The plates were incubated at $35^{\circ} \mathrm{C}$ for $48 \mathrm{~h}$. Yeast growth was visually evaluated, and the MICs for the azole derivatives and caspofungin were determined as the lowest concentration able of reducing $50 \%$ of yeast growth, when compared to drug-free growth control, while the MICs for amphotericin B were determined as the lowest concentration capable of inhibiting $100 \%$ of fungal growth. MIC values $\geq 64$ and $\geq 1 \mu \mathrm{g} / \mathrm{mL}$ indicated in vitro resistance to fluconazole and itraconazole, respectively. For the species C.parapsilosis and C. tropicalis, MICs $\geq 8 \mu \mathrm{g} / \mathrm{mL}$ for fluconazole indicated resistance (CLSI, 2012). For caspofungin, MIC values $\geq 0.5 \mu \mathrm{g} / \mathrm{mL}$ against $C$. glabrata, $\geq 1 \mu \mathrm{g} / \mathrm{mL}$ against $C$. tropicalis, and C. krusei and $\geq 8 \mu \mathrm{g} / \mathrm{mL}$ against $C$. parapsilosis and C. guilliermondii indicated resistance. ${ }^{17}$ For the other Candida species, caspofungin MIC values $>2 \mu \mathrm{g} / \mathrm{mL}$ indicated that the strain is not susceptible to this drug. ${ }^{16}$

\section{Antifungal susceptibility of mature Candida biofilms}

The method described by Ramage et al. ${ }^{18}$ with modifications, was used to assess the inhibitory effect of the antifungal drugs on the biofilms formed by the tested strains. Biofilms were produced as described above. Afterward, the medium was aspirated and the nonadhered cells were removed by three washes with sterile PBS. The residual PBS was completely removed and the plate was dried with paper towel, before adding $100 \mu \mathrm{L}$ of the drugs amphotericin B, fluconazole, itraconazole, and caspofungin, at two concentrations, 10xMIC and 50xMIC, based on the MICs obtained against each strain in planktonic form. After the addition of $100 \mu \mathrm{L}$ of each drug, the plates were further incubated at $35^{\circ} \mathrm{C}$, for $48 \mathrm{~h}$.
The viability of the mature biofilms was assessed by the XTT assay, according to Sidrim et al. ${ }^{19}$ XTT is an oxidoreduction indicator that is reduced by mitochondrial dehydrogenases of metabolically active cells to the watersoluble formazan product. This product is spectrophotometrically measured, thus, allowing the quantification of cell metabolism and biofilm metabolic activity. This is essential for biofilm susceptibility assays, as the viability of these fungal structures cannot be visually assessed, as described for the antifungal susceptibility assays of planktonic fungal growth. ${ }^{18}$ The XTT stock solutions $(1 \mathrm{mg} /$ $\mathrm{mL}$ ) were previously prepared, filtered, and stored at $-20{ }^{\circ} \mathrm{C}$ until use. Menadione (Sigma) $(0.4 \mathrm{mM}$ in acetone), prepared at the moment of use, was employed as an electron-coupling agent. Aliquots of $50 \mu \mathrm{L}$ of sterile PBS, $75 \mu \mathrm{L}$ of XTT solution, and $6 \mu \mathrm{L}$ of menadione solution were added to each of the wells containing biofilms. The plates were incubated at $35^{\circ} \mathrm{C}$ for $5 \mathrm{~h}$ in the dark. After this period, XTT solution was transferred to a new flatbottom 96-well plate and spectrophotometrically read, at $492 \mathrm{~nm}$. All tests were run in triplicate, and drug-free growth control and yeast-free sterility control were included.

\section{Statistical methods}

Regarding the biofilm assay, all tests were performed in triplicate and the results of the absorbance of the XTT assay were evaluated by ANOVA and Tukey's multiple comparisons post-test. $P$-values $<0.05$ were considered to be statistically significant.

\section{RESULTS}

Biofilm formation was evaluated for all 15 strains, of which eight (3/4 C. tropicalis; $1 / 3$ C. parapsilosis sensu stricto; 2/2 C. glabrata; $1 / 1$ C. guilliermondii and 1/1 C. catenulata) were able to form biofilms (Table 1). One strain was classified as a strong biofilm producer (OD590 nm $>1.908$, Fig. 1a), three as moderate producers $(0.954<\mathrm{O}$ D590 nm $\leq 1.908$, Fig. 1b), and four as weak producers $(0.477<$ OD590 $\mathrm{nm} \leq 0.954$, Fig. 1c), as reported in Table 1. As shown in the SEM analysis, C. tropicalis and C. guilliermondii biofilms are formed by layers of blastoconidia and pseudohyphae, and C. glabrata biofilms are formed by layers of blastoconidia only (Fig. 1).

Regarding antifungal susceptibility, the antifungal MICs against Candida strains in planktonic form were visually determined, through broth microdilution. The obtained MICs varied from 0.03125 to $1 \mu \mathrm{g} / \mathrm{mL}$ for amphotericin $\mathrm{B}$, from 0.03125 to $>16 \mu \mathrm{g} / \mathrm{mL}$ for itraconazole, from 0.25 to $>64 \mu \mathrm{g} / \mathrm{mL}$ for fluconazole, and from 0.03125 to $1 \mu \mathrm{g} / \mathrm{mL}$ for caspofungin (Table 2). Two strains of C. tropicalis and one of C. catenulata were resistant to itraconazole (MIC $\geq 1 \mu \mathrm{g} / \mathrm{mL}$ ), while only one strain of C. parapsilosis sensu stricto was resistant to fluconazole (MIC $\geq 8 \mu \mathrm{g} / \mathrm{mL}$ ). One strain of $C$. glabrata was resistant 
Table 1. Biofilm production of Candida species recovered from the ocular conjunctiva of horses and antifungal susceptibility of the strains in planktonic form

\begin{tabular}{|c|c|c|c|c|c|c|}
\hline \multirow[b]{2}{*}{ Strains } & \multirow[b]{2}{*}{ Candida species } & \multirow[b]{2}{*}{ Biofilm production } & \multicolumn{4}{|c|}{ Minimum inhibitory concentration $(\mu \mathrm{g} / \mathrm{mL})$} \\
\hline & & & Amphotericin B & Fluconazole & Itraconazole & Caspofungin \\
\hline 01 & C. tropicalis & Absent & 1 & 1 & 0.25 & 0.03125 \\
\hline 02 & C. tropicalis & Moderate & 1 & 1 & $1^{*}$ & 0.03125 \\
\hline 03 & C. tropicalis & Strong & 1 & 0.5 & $4 *$ & 0.0625 \\
\hline 04 & C. tropicalis & Moderate & 1 & 1 & 0.5 & 0.125 \\
\hline 05 & C. parapsilosis & Absent & 0.25 & $16^{*}$ & 0.0625 & 0.03125 \\
\hline 06 & C. parapsilosis & Weak & 0.25 & 0.5 & 0.03125 & 1 \\
\hline 07 & C. parapsilosis & Absent & 0.03125 & 2 & 0.03125 & 0.03125 \\
\hline 08 & C. glabrata & Weak & 1 & $64 *$ & $16^{*}$ & 0.03125 \\
\hline 09 & C. glabrata & Moderate & 1 & 2 & $1^{*}$ & $0.5^{*}$ \\
\hline 10 & C. rugosa & Absent & 0.5 & 4 & 0.5 & 0.0625 \\
\hline 11 & C. famata & Absent & 1 & 4 & 0.25 & 0.0625 \\
\hline 12 & C. ciferrii & Absent & 0.5 & 2 & 0.0625 & 0.0625 \\
\hline 13 & C. guilliermondii & Weak & 1 & 0.25 & 0.0625 & 0.03125 \\
\hline 14 & C. catenulata & Weak & 0.5 & 4 & $1 *$ & 0.25 \\
\hline 15 & C. krusei & Absent & 1 & 0.25 & 0.03125 & $1 *$ \\
\hline
\end{tabular}

Antifungal minimum inhibitory concentrations against planktonic Candida spp. were determined through broth microdilution (CLSI, document M27-A3). *Antifungal resistance (fluconazole: $\geq 8 \mu \mathrm{g} / \mathrm{mL}$ against C. parapsilosis and C. tropicalis, $\geq 64 \mu \mathrm{g} / \mathrm{mL}$ against $C$. glabrata; itraconazole: $\geq 1 \mu \mathrm{g} / \mathrm{mL}$; caspofungin: $\geq 8 \mu \mathrm{g} / \mathrm{mL}$ against $C$. parapsilosis and $C$. guilliermondii, $\geq 1 \mu \mathrm{g} / \mathrm{mL}$ against C. tropicalis and C. krusei, $\geq 0.5 \mu \mathrm{g} / \mathrm{mL}$ against C. glabrata).

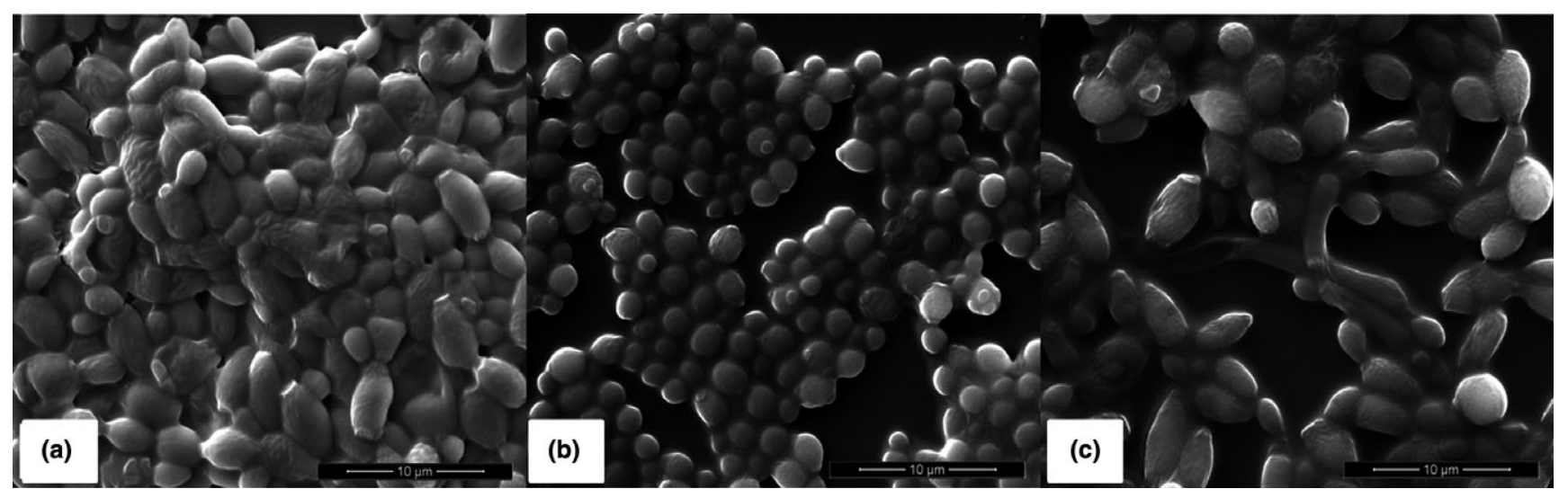

Figure 1. Mature biofilms of Candida spp. (a) C. tropicalis with strong biofilm production (OD590 nm $>1.908$ ). Notice the multilayer arrangement of blastoconidia and pseudohyphae; (b) C. glabrata with moderate biofilm production $(0.954<$ OD590 nm $\leq 1.908)$. Notice the clusters of small round blastoconidia, in a monolayer arrangement, and the absence of pseudohyphae, with empty areas on the coverslide; (c) C. guilliermondii with weak biofilm production $(0.477<$ OD590 nm $\leq 0.954)$. Notice clusters of blastoconidia and pseudohyphae, in a monolayer arrangement, with empty areas on the coverslide.

to both fluconazole and itraconazole, with MICs $\geq 64 \mu \mathrm{g} /$ $\mathrm{mL}$ and $1 \mu \mathrm{g} / \mathrm{mL}$, respectively, and another one was simultaneously resistant to itraconazole $(\mathrm{MIC}=1 \mu \mathrm{g} / \mathrm{mL})$ and caspofungin $(\mathrm{MIC}=0.5 \mu \mathrm{g} / \mathrm{mL})$. Resistance to caspofungin was also observed in one strain of C. krusei $(\mathrm{MIC}=1 \mu \mathrm{g} / \mathrm{mL})$. The antifungal susceptibility results are shown in Table 1 .

In order to assess biofilm antifungal susceptibility, the eight biofilm-producing strains were used, and mature biofilms were exposed to two antifungal concentrations, 10xMIC and 50xMIC, based on the MICs obtained against each strain in planktonic form. Considering that the effect of antifungal drugs on biofilms cannot be visually read, as described for broth microdilution, the biofilm metabolic activity, after exposure to antifungals, was measured through XTT assay, in order to assess biofilm viability. Thus, the biofilm metabolic activity of one strain of $C$. tropicalis did not reduce after exposure to any of the tested antifungal drugs. Five strains (two C. tropicalis, one C. glabrata, one C. guilliermondii, and one C. catenulata) presented significant reduction in biofilm metabolic activity, when compared to the growth control $(P<0.05)$, only after exposure to amphotericin B at 50xMIC. The other tested drugs (azoles and caspofungin) presented no inhibitory effects on the biofilm of these strains. Finally, two strains (one C. parapsilosis sensu stricto 


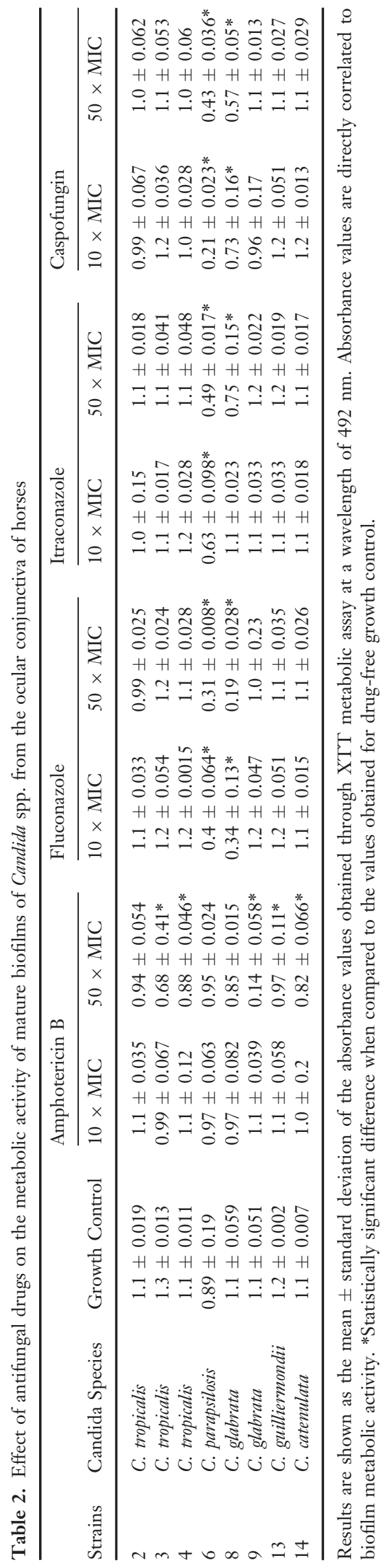

and one C. glabrata) did not show reduction in biofilm metabolic activity after exposure to amphotericin B at either of the tested concentrations. However, biofilm metabolic activity of the C. parapsilosis sensu stricto strain significantly declined $(P<0.05)$ in the presence of fluconazole, itraconazole, and caspofungin, at both tested concentrations. As for the C. glabrata strain, a significant reduction in biofilm metabolic activity was observed at both tested concentrations of fluconazole $(P<0.05)$ and caspofungin $(P<0.05)$, and only at 50xMIC of itraconazole $(P<0.05)$ (Table 2$)$.

\section{DISCUSSION}

The tested species of the Candida genus have been described as components of the microbiota of several animal species ${ }^{3,20-25}$ and pathogenic agents causing keratitis in horses. ${ }^{4-7}$ Studies on the conjunctival microbiota of healthy horses are scarce, but they are important because the fungal composition may be influenced by geographic region. ${ }^{26}$ Some authors have reported the isolation of Candida spp., but the species were not determined., ${ }^{2,3,27}$ The strains used in this research were recovered from healthy horses, providing data on the yeast composition of the conjunctival microbiota.

Fungal infections are a common cause of corneal diseases in horses, accounting for up to one-third of all inflammatory keratopathies in this animal species. ${ }^{28}$ Keratomycosis is a sight-threatening, frequently painful condition that can result in vision or eye globe loss, often requiring an aggressive and long-lasting treatment. ${ }^{4,26,29}$ The high incidence of corneal infections in horses is mainly associated with the large size of the eye globe with large corneal surface area; the prominent lateral position of the eye; low resting temperature of the cornea; and the presence of fungi in the conjunctival microbiota and in the environment where the animals are kept. ${ }^{1,4}$ Keratomycosis is a common infection in equines because the tear film of these animals has weak immunoprotective properties. $^{4,27}$

In addition to these anatomical and physiological predisposing features of the equine eye, some specific conditions also contribute for the development of keratomycosis in these animals. For instance, corneal epithelial injuries or alterations of the tear film allow the entrance of opportunistic fungi from the conjunctival microbiota or the environment into the corneal tissue. ${ }^{4,28}$ These fungi may colonize the cornea, penetrate its stroma, and migrate between its collagen layers. ${ }^{30}$ Fungal invasion triggers an inflammatory response and protease overproduction, leading to corneal stromal defects, which may progress to corneal perforation. ${ }^{28}$ The most common fungi associated with cases of equine keratomycosis are Aspergillus spp., Fusarium spp., and Candida spp. ${ }^{7,29}$

The pathogenicity of Candida species is mediated by several virulence factors, including biofilm formation on 
biotic and abiotic surfaces, ${ }^{8}$ which is associated with the development of antimicrobial resistance. ${ }^{12}$ In veterinary medicine, the presence of microbial biofilms has also been associated with antimicrobial resistance and clinical relapses. The importance of biofilm infections in animals still needs to be addressed, but evidences show that several bacterial infections are associated with biofilm growth, including pneumonia, liver abscesses, enteritis, otitis, mastitis, and wound infections, ${ }^{9}$ especially in horses. ${ }^{31}$ On the other hand, little is known on the biofilm-forming ability of fungal pathogens from animals and its association with the development of diseases. For instance, it has been shown that veterinary strains of Malassezia pachydermatis, ${ }^{32}$ C. parapsilosis sensu lato, ${ }^{33}$ and C. tropicalis $^{34}$ are capable of producing biofilms in vitro.

In our study, $53.3 \%$ of the strains from the ocular conjunctiva of horses showed the ability to form biofilms, as evidenced by the crystal violet technique, which quantifies microbial biofilm-forming ability through the amount of dye retained by the yeast cells and extracellular polymeric substances adhered to the walls of a polystyrene plate. ${ }^{13,14}$ Two C. tropicalis strains were characterized as moderate producers and one as a strong producer. Some studies have shown that $C$. tropicalis has a remarkable biofilmforming ability, ${ }^{8,34}$ and Cordeiro et al., ${ }^{34}$ for instance, have shown that $C$. tropicalis isolated from animals has a noteworthy in vitro biofilm production. Additionally, two strains of $C$. glabrata were able to produce biofilm, and were classified as weak and moderate producers, corroborating the findings of Silva et al., ${ }^{8}$ who reported a weaker biofilm-forming ability for this species.

Several studies have reported that biofilms formed by Candida spp. are resistant to antifungal drugs commonly used in clinical practice, especially azole derivatives. ${ }^{35,36}$ Our results corroborate these observations, once Candida biofilms were able to tolerate higher concentrations of antifungal drugs, when compared to their planktonic counterparts, as demonstrated by the XTT assay, which detects biofilm metabolic activity, hence, allowing the assessment of biofilm viability and its susceptibility to antifungal drugs. ${ }^{18,19}$ In the present study, only one strain of $C$. parapsilosis sensu stricto and one C. glabrata were inhibited by itraconazole and fluconazole, while the other tested strains tolerated both azole drugs, even at 50xMIC. Similarly, Brilhante et al. ${ }^{37}$ demonstrated that C. tropicalis biofilms are not affected by very high concentrations of azole drugs. Some hypotheses have been proposed to explain the reduced susceptibility of Candida biofilms to azole drugs, including the reduced metabolism of biofilm cells, decreased drug penetration through the biofilm matrix, and increased expression of specific genes by biofilm cells, especially those that encode efflux pumps. ${ }^{38,39}$

Regarding the inhibitory activity of amphotericin B, five of the eight tested biofilm-producing strains presented a reduction in biofilm metabolic activity, after exposure to this drug, corroborating the findings of Brucker et al. ${ }^{40}$ and Brilhante et al., ${ }^{37}$ who reported that amphotericin B has a consistent activity against biofilms of Candida spp. Unlike azole derivatives, whose inhibitory activity depends on the metabolism of fungal cells, more specifically on ergosterol synthesis, amphotericin B exerts its activity through hydrophobic interactions with preformed ergosterol molecules on cell membrane, disrupting membrane function. ${ }^{41}$ Therefore, amphotericin B does not need metabolically active cells to exert its inhibitory effect, which may explain its better activity against Candida biofilms, when compared to azole drugs.

Caspofungin plays an important role in the treatment of infections caused by different azole-resistant fungi. ${ }^{42}$ Moreover, it has been described to have a remarkable activity against C. albicans $^{36}$ and C. tropicalis $^{37}$ biofilms. However, in our study, this drug only significantly reduced the biofilm metabolic activity of one $C$. parapsilosis sensu stricto and one C. glabrata.

Our results demonstrate that the isolates from the conjunctival microbiota of horses were able to produce biofilms, which presented reduced susceptibility to the four tested antifungal drugs. This fact highlights the potential involvement of Candida spp. from the conjunctival microbiota of horses in keratomycoses, as these infections are often associated with biofilm growth on and within corneal tissue. ${ }^{11}$ Therefore, even though the tested strains were recovered from healthy horses, it is important to highlight that Candida species are opportunistic pathogens that may become pathogenic, under certain circumstances, ${ }^{4,28}$ emphasizing the importance of assessing their biofilm-producing ability and antifungal susceptibility. Finally, further studies comparing Candida spp. from healthy and diseased horses with keratomycosis may be beneficial to elucidate other pathophysiological aspects of Candida corneal infections.

In summary, the data from this study show that the Candida species isolated from the ocular conjunctiva of horses can pose a risk to animals' health, as they are capable of forming biofilms, which are commonly involved in fungal keratitis.

\section{ACKNOWLEDGMENTS}

This work was supported by grants from the National Council for Scientific and Technological Development (CNPq; Brazil; 307606/2013-9; 443167/2014-1).

\section{CONFLICT OF INTEREST}

None.

\section{REFERENCES}

1. Galera PD, Brooks DE. Optimal management of equine keratomycosis. Veterinary Medicine: Research and Reports 2012; 3: $7-17$. 
2. Barsotti G, Sgorbini M, Nardoni S et al. Occurrence of fungi from conjunctiva of healthy horses in Tuscany, Italy. Veterinaty Research Communication 2006; 30: 903-906.

3. Souza ME, Araújo MAS, Mota RA et al. Fungal microbiota from ocular conjunctiva of clinically healthy horses belonging to the military police cavalry of Alagoas. Brazilian Fournal of Microbiology 2011; 42: 1151-1155.

4. Voelter-Ratson K, Pot SA, Florin M et al. Equine keratomycosis in Switzerland: a retrospective evaluation of 35 horses (January 2000-August 2011). Equine Veterinary fournal 2013; 45: 608-612.

5. Ledbetter EC, Irby NL, Kim SG. In vivo confocal microscopy of equine fungal keratitis. Veterinary Opbthalmology 2011; 14: 1-9.

6. Brooks DE, Plummer CE, Mangan BG et al. Equine subepithelial keratomycosis. Veterinary Ophthalmology 2013; 16: 93-96.

7. Ledbetter EC, Patten VH, Scarlett JM et al. In vitro susceptibility patterns of fungi associated with keratomycosis in horses of the northeastern United States: 68 cases (1987-2006). Fournal of the American Veterinary Medical Association 2007; 231: 1086-1091.

8. Silva S, Negri M, Henriques M et al. Candida glabrata, Candida parapsilosis and Candida tropicalis: biology, epidemiology, pathogenicity and antifungal resistance. FEMS Microbiology Review 2012; 36: 288-305.

9. Clutterbuck AL, Woods EJ, Knottenbelt DC et al. Biofilms and their relevance to veterinary medicine. Veterinary Microbiology 2007; 121: 1-17.

10. Ramage G, Rajedran R, Sherry L et al. Fungal biofilm resistance. International fournal of Microbiology 2012; 2012: doi: 10.1155/ 2012/528521.

11. Liu L, Wu H, Riduan SN et al. Short imidazolium chains effectively clear fungal biofilm in keratitis treatment. Biomaterials 2013; 34: 1018-1023.

12. Sardi JCO, Scorzoni L, Bernardi T et al. Candida species: current epidemiology, pathogenicity, biofilm formation, natural antifungal products and new therapeutic options. Fournal of Medical Microbiology 2013; 62: 10-24.

13. Chatzimoschou A, Katragkou S, Simitsopoulou M et al. Activities of triazole-echinocandin combinations against Candida species in biofilms and as planktonic cells. Antimicrobial Agents and Chemotherapy 2011; 55: 1968-1974.

14. Stepanovic S, Vukovic D, Dakic I et al. A modified microtiterplate test for quantification of staphylococcal biofilm formation. Fournal of Microbiological Methods 2000; 40: 175-179.

15. Wang WL, Yang RY, Ao JH. Uncommon characteristics of the structure and development of Trichosporon asabii. Chinese Medical fournal 2009; 122: 1806-1810.

16. Clinical Laboratory Standards Institute, 2008. Reference method for broth dilution antifungal susceptibility testing of yeasts. Approved standard M27-A3. National Committee for Clinical Laboratory Standards, Wayne, Pa.

17. Clinical Laboratory Standards Institute, 2012. Reference method for broth dilution antifungal susceptibility testing of yeasts. Approved standard M27-S4. National Committee for Clinical Laboratory Standards, Wayne, Pa.

18. Ramage G, Walle KV, Wickes BL et al. Standardized method for in vitro antifungal susceptibility testing of Candida albicans biofilms. Antimicrobial Agents and Chemotherapy 2001; 45: $2475-$ 2479 .

19. Sidrim JJC, Teixeira CEC, Cordeiro RA et al. $\beta$-Lactam antibiotics and vancomycin inhibit the growth of planktonic and biofilm Candida spp.: an additional benefit of antibiotic-lock therapy? International fournal of Antimicrobial Agents 2015; 45: $420-423$.

20. Santos RC, Marin JM. Isolation of Candida spp. from mastitic bovine milk in Brazil. Mycopathologia 2005; 159: 251-253.

21. Sgorbini M, Barsotti G, Nardoni S et al. Fungal flora of normal eyes in healthy newborn foals living in the same stud farm in Italy. Fournal of Equine Veterinary Science 2008; 28: 540-543.

22. Brilhante RSN, Paiva MAN, Sampaio CMS et al. Yeasts from Macrobrachium amazonicum: a focus on antifungal susceptibility and virulence factors of Candida spp. FEMS Microbiology Ecology 2011; 76: 1-10.

23. Brilhante RSN, Castelo-Branco DSC, Duarte GPS et al. Yeast microbiota of raptors: a possible tool for environmental monitoring. Environmental Microbiology Reports 2012; 4: 189-193.

24. Brilhante RSN, Alencar LP, Cordeiro RA et al. Detection of Candida species resistant to azoles in the microbiota of rheas (Rhea americana): possible implications for human and animal health. Fournal of Medical Microbiology 2013; 62: 889-895.

25. Cordeiro RA, Bittencourt PV, Brilhante RSN et al. Species of Candida as a component of the nasal microbiota of healthy horses. Medical Mycology 2013; 51: 731-736.

26. Voelter-Ratson K, Monod M, Unger L et al. Evaluation of the conjunctival fungal flora and its susceptibility to antifungal agents in healthy horses in Switzerland. Veterinary Opbthalmology 2014; 17: 31-36.

27. Araghi-Sooreh A, Navidi M, Razi M. Conjunctival bacterial and fungal isolates in clinically healthy working horses in Iran. Kafkas Universitesi Veteriner Fakultesi Dergisi 2014; 20: 625-627.

28. Sherman $A B$, Clode $A B$, Gilger $B C$. Impact of fungal species cultured on outcome in horses with fungal keratitis. Veterinary Ophthalmology 2016; 20: 140-146. doi: 10.1111/vop.12381. [ahead of print]

29. Reed Z, Thomasy SM, Good KL et al. Equine keratomycoses in California from 1987 to 2010 (47 cases). Equine Veterinary Fournal 2012; 45: 361-366.

30. Galera PD, Martins BC, Laus JL et al. Ceratomicose em eqüinos. Ciência Rural 2012; 42: 1223-1230.

31. Westgate SJ, Percival SL, Knottenbelt DC et al. Microbiology of equine wounds and evidence of bacterial biofilms. Veterinary Microbiology 2011; 150: 152-159.

32. Figueredo LA, Cafarchia C, Desantis S et al. Biofilm formation of Malassezia pachydermatis from dogs. Veterinary Microbiology 2012; 160: 126-131.

33. Brilhante RSN, Rodrigues TJS, Castelo-Branco DSCM et al. Antifungal susceptibility and virulence attributes of animalderived isolates of Candida parapsilosis complex. Fournal of Medical Microbiology 2014; 63: 1568-1572.

34. Cordeiro RA, Oliveira JS, Castelo-Branco DSCM et al. Candida tropicalis isolates obtained from veterinary sources show resistance to azoles and produce virulence factors. Medical Mycology 2015; 53: $145-152$.

35. Shinde RB, Chauhan NM, Raut JS et al. Sensitization of Candida albicans biofilms to various antifungal drugs by cyclosporine A. Annals of Clinical Microbiology and Antimicrobials 2012; doi: 10. 1186/1476-0711-11-27.

36. Sarkar S, Uppuluri P, Pierce CG et al. In vitro study of sequential fluconazole and caspofungin treatment against Candida albicans biofilms. Antimicrobial Agents and Chemotherapy 2014; 58: 1183-1186.

37. Brilhante RS, Oliveira JS, Evangelista AJ et al. Candida tropicalis from veterinary and human sources shows similar in vitro hemolytic activity, antifungal biofilm susceptibility and 
pathogenesis against Caenorbabditis elegans. Veterinary Microbiology 2016; 192: 213-219.

38. Vediyappan G, Rossignol T, D'Enfert C. Interaction of Candida albicans biofilms with antifungals: transcriptional response and binding of antifungals to beta-glucans. Antimicrobial Agents and Chemotherapy 2010; 54: 2096-2111.

39. Mitchell KF, Taff HT, Cuevas MA et al. Role of matrix $-1,3$ glucan in antifungal resistance of non-albicans Candida biofilms. Antimicrobial Agents and Chemotherapy 2013; 57: 19181920.
40. Brucker K, Bink A, Meert E et al. Potentiation of antibiofilm activity of amphotericin B by superoxide dismutase inhibition. Oxidative Medicine and Cell Longevity 2013; 2013: doi: 10.1155/ 2013/704654.

41. Nett JE, Andes DR. Antifungal agents: spectrum of activity, pharmacology and clinical indications. Infectious Disease Clinics of North America 2016; 30: 51-83.

42. Bachmann SP, Walle KV, Ramage $\mathrm{G}$ et al. In vitro activity of caspofungin against Candida albicans biofilms. Antimicrobial Agents and Chemotherapy 2002; 46: 3591-3596. 